\title{
Electromagnetic ion-cyclotron instability in the presence of a parallel electric field with general loss-cone distribution function - particle aspect analysis
}

\author{
G. Ahirwar, P. Varma, and M. S. Tiwari \\ Department of Physics \& Electronics, Dr. H. S. Gour University, Sagar (M.P.) - 470003, India \\ Received: 6 March 2006 - Revised: 18 May 2006 - Accepted: 23 May 2006 - Published: 9 August 2006
}

\begin{abstract}
The effect of parallel electric field on the growth rate, parallel and perpendicular resonant energy and marginal stability of the electromagnetic ion-cyclotron (EMIC) wave with general loss-cone distribution function in a low $\beta$ homogeneous plasma is investigated by particle aspect approach. The effect of the steepness of the loss-cone distribution is investigated on the electromagnetic ion-cyclotron wave. The whole plasma is considered to consist of resonant and nonresonant particles. It is assumed that resonant particles participate in the energy exchange with the wave, whereas nonresonant particles support the oscillatory motion of the wave. The wave is assumed to propagate parallel to the static magnetic field. The effect of the parallel electric field with the general distribution function is to control the growth rate of the EMIC waves, whereas the effect of steep loss-cone distribution is to enhance the growth rate and perpendicular heating of the ions. This study is relevant to the analysis of ion conics in the presence of an EMIC wave in the auroral acceleration region of the Earth's magnetoplasma.
\end{abstract}

Keywords. Magnetospheric physics (Auroral phenomena; Electric fields) - Space plasma physics (Wave-particle interactions)

\section{Introduction}

The currents in the auroral ionosphere are part of a large, three-dimensional current system, coupling the ionosphere to magnetosphere. One of the main problems in auroral plasma physics concerns the acceleration of the electrons to kinetic energies much higher than their initial thermal energies. Both theory and observations from both the current regions downward (Marklund et al., 2001) and upward (McFadden et al.,

Correspondence to: M. S. Tiwari

(tiwarims@yahoo.co.in)
1999) have indicated that the electrons are accelerated by parallel electric fields.

The precipitating electrons cause the aurora and carry the field-aligned currents in the upward current regions. To reach the ionosphere, the electrons must have a pitch angle, defined as $\theta=\arctan \left(\frac{v_{\perp}}{v_{U}}\right)$, where $v_{\perp}$ and $v_{\amalg}$ are the perpendicular and parallel components of the electron thermal velocity, which is within the so-called loss cone, defined as $\theta_{l c}=\arcsin \left[\left(\mathrm{B}_{2}\right.\right.$ $\left./ \mathrm{B}_{1}\right)^{1 / 2}$, where $B_{2}$ is the magnetic field at a certain altitude and $\mathrm{B}_{1}$ is the magnetic field strength in the ionosphere. The importance of the parallel electric fields is well established (Evans, 1968; Mozer and Fahleson, 1970; Gurnett and Frank, 1973; Mozer and Hull, 2001; Hull et al., 2003) and the theories regarding the origin of the parallel electric field forming the aurora have numbered at least 22, according to the review study by Borovsky (1993). However, a collisionless plasma has a high parallel conductivity and any parallel electric field should be short-circuited. Some theories show that parallel electric fields can exist in spite of the high conductivity. A simple theory is that when a current is drawn through lowdensity plasma above the ionosphere, parallel electric fields form and accelerate the electrons to keep the current continuity (Ronnmark, 1999). Another theory is that parallel electric fields are associated with shear Alfvén waves (Goertz and Bosewell, 1979; Lysak and Dum, 1983; Genot et al., 1999; Ronnmark and Hamrin, 2000). An additional accelerating mechanism is parallel electric fields set up because of anomalous resistivity caused by waves (Papadopolous, 1977; Hudson, 1978; Lysak and Hudson, 1979).

Parallel electric fields are known to be a primary acceleration mechanism in the upward current region of the aurora. They have been inferred from observations on sounding rockets and satellites (Evans, 1974; Mozer et al., 1977) and direct measurements have been reported (Mozer and Kletzing, 1998). The first direct electric field evidence of parallel electric fields in the auroral acceleration region was

Published by Copernicus GmbH on behalf of the European Geosciences Union. 
made on the S3-3 satellite through observation of very large $(\sim 1000 \mathrm{mV} / \mathrm{m})$ perpendicular electric fields, called electrostatic shocks (Mozer et al., 1977). The S3-3 satellite also made direct measurements of parallel electric fields of hundreds of $\mathrm{mV} / \mathrm{m}$ (Mozer et al., 1980). Observations from the FAST satellite (Chaston et al., 1999, 2000, 2002a, b) have indicated that precipitating auroral electrons often are field aligned and have a broad distribution in energy, in contrast to the typical auroral "inverted" type precipitation, which have a characteristic energy but are broad in pitch angle. Regions of this type of field-aligned acceleration have been observed throughout the auroral zone but are often seen at the polar cap boundary of the auroral zone. Similar observations of field-aligned electrons have been seen over many years, particularly from sounding rocket missions (Johnstone and Winningham, 1982; Arnoldy et al., 1985; McFadden et al., 1998; Robinson et al., 1989; Knudsen et al., 1998). These fieldaligned distributions are not consistent with the usual picture of a plasma sheet distribution that has been accelerated in a quasi-static potential drop. Rather, it has been suggested that the low-frequency waves responsible for this acceleration are Alfvénic or EMIC waves (Kletzing, 1994; Thompson and Lysak, 1996; Chaston et al., 1999, 2000, 2002a, b; Kletzing and $\mathrm{Hu}, 2001)$. An enhancement of the abundance of cold plasma in the ring current can cause the growth of the electromagnetic ion cyclotron (EMIC) instability, forcing ring current ions into the loss cone. Recent modeling of large magnetospheric storms shows that EMIC waves can contribute significantly to ion precipitation in the afternoon local time sector (Jordanova et al., 2001).

The importance of ion cyclotron waves in auroral physics lies in their ability to heat ions, accelerate/modulate electrons, and perhaps to provide anomalous resistivity, allowing for the creation of a parallel potential drop. Since such processes have global implications for the magnetosphere, these waves have been the subject of numerous publications (Temerin and Lysak, 1984; McFadden et al., 1998a; Erlandson and Zanetti, 1998). Observations from the Fast Auroral Snapshot (FAST) spacecraft at $\sim 4000 \mathrm{~km}$ altitude in ion beam regions (Cattell et al., 1998; Ergun et al., 1998a; McFadden et al., 1998a) shows that these waves are electromagnetic. Chaston et al. (1998) have shown that these waves can have Poynting fluxes directed upward out of the auroral oval, suggesting that the waves observed at higher altitudes may have their source at the base of the auroral potential structure where they are electromagnetic.

In 1977, Sharp et al. (19977) first deduced the existence of a mechanism that accelerations of ions transversely to the magnetic field at auroral latitudes, ion conics have come to be recognized as ubiquitous features of the aurora. A large number of rocket (Wahlen et al., 1978; Yau et al., 1983; Kintner et al., 1986; Lynch et al., 1996) and satellite (Gorney et al., 1981; Klumpar et al., 1984; Miyake et al., 1991, 1996; Kundsen et al., 1998a; Andre et al., 1998) studies have attempted to elucidate the mechanisms which produce trans- verse ion acceleration. In recent years three types of wave emissions have been found in association with ion conics at altitudes below $2000 \mathrm{~km}$. The most common is a broadband, extremely low frequency (BBELF) emission, which is correlated with field-aligned suprathermal electron bursts in the downward current region (Kundsen et al., 1998a). The other two types of wave emissions, electromagnetic ion cyclotron (EMIC) waves (Erlandson et al., 1996) and lower hybrid (LH) waves (Kintner et al., 1986), are associated with field-aligned electrons occurring within "inverted-V" structures. A recent statistical survey of the Freja data confirm that these wave emissions account for nearly all of the ion conics seen near $1700 \mathrm{~km}$ (Andre et al., 1998).

In this paper, we present a systematic and detailed investigation of EMIC instabilities with parallel electric field and loss-cone distribution functions for magnetospheres like plasma parameters, with the purpose of attaining a more complete understanding of their relative importance. In most of the theoretical work reported so far, the velocity distribution function has been assumed to be either Maxwellian or biMaxwellian, ignoring the steep loss-cone feature. Plasma in mirror-like devices and in the auroral region with curved and converging magnetic field lines, depart considerably from the Maxwellian distribution and have a steep loss-cone distribution (Tiwari and Varma, 1991; Dwivedi et al., 2001a, b; Misra and Tiwari, 1979), provided there is a relatively low degree of plasma collisionality.

The method adopted known as particle analysis had been widely used to the analysis of electrostatic and electromagnetic instabilities (Tiwari and Varma,1991; Dwivedi et al., 2001a, b, 2002; Duan et al., 2005; Mishra and Tiwari, 2006). The relative importance of this approach over fluid and kinetic approachs is also discussed (Varma and Tiwari, 1992, 1993; Tiwari and Varma, 1993).

The main advantages of this approach are to consider the energy transfer between wave and particles, along with the discussion of wave dispersion and the growth/damping rate of the wave. The method may be suitable to deal with the auroral electrodynamics, where particle acceleration is also important along with wave emissions. The results obtained by this approach are the same as those derived using the kinetic approach.

Effects of the general distribution function with the parallel electric field have been widely studied by a number of workers concerning electrostatic waves, electromagnetic waves, drift waves, Alfvén and kinetic Alfvén waves, and electrostatic ion cyclotron waves (Tiwari and Varma, 1991; Bajaj and Tiwari, 1992; Dwivedi et al., 2001a, b; Mishra and Tiwari, 2006).

In this paper the general loss-cone distribution function with the large parallel electric field is used to study EMIC waves. The wave propagating in the direction of the ambient magnetic field along the $\mathrm{z}$-axis is considered. The whole plasma has been considered to consist of resonant and nonresonant particles. The resonant particles participate in an 
energy exchange process, whereas the non-resonant particles support the oscillatory motion of the wave. The organization of the paper is as follows. An introduction is given in Sect. 1 . EMIC wave observations are given in Sect. 2. The detail mathematical analysis is in Sect. 3. In Sect. 4, we placed the results and discussions obtained by investigation.

\section{Observations}

Observational evidence of EMIC waves in the auroral acceleration region are presented from the FAST satellites (Chaston et al., 2002a, b) and large parallel electric fields measured by the Duo Deca Electron Ion Spectrometer (DDEIS) component of the HYDRA instruments on board Polar satellites (Scudder et al., 1995; Hull et al., 2003). These evidences are considered in this model, which has calculated the effect of the parallel electric field on the EMIC wave with loss-cone distribution function by particle aspect approach. Here we see that these theoretical results are predicted by mathematical evaluation and graphical presentation with the growth of the EMIC wave with the parallel electric field in the auroral acceleration region, which is observationally shown by Freja and FAST satellites (Mozer and Hull, 2001; Lund et al., 2000). Lund et al. (2000) predict the distribution of EMIC ion conics, which are similar to that of auroral EMIC waves (Saito et al., 1987). The EMIC ion conics, with phenomena that are associated with parallel electric fields, suggest that parallel electric fields play a significant role in transverse ion heating in the aurora. It is predicted that the observational results give us good agreement with our theoretical model.

As the statistical study described below shows in Fig. 1 (Lund et al., 2000), almost all of the ion conic events seen by FAST are associated with emissions of EMIC waves. Figure 1 shows the example of transverse ion heating by EMIC waves. These data are taken from a pass near 22:00 MLT. This pass was geomagnetically quiet $\left(K_{p}-1-\right)$. The VLF portion of the wave spectrum shows evidence of a particularly low plasma density on this pass (Strangeway et al., 1998). The ion conic event occurs at 06:44:44-58, between two ion beams; many of the EMIC conics occur at the edge of an ion beam. The EMIC waves occur in the presence of secondary electrons, which are the leading candidate for generating the waves.

Figure 2 shows (Lund et al., 2000) the distribution of events in latitude and magnetic local time. In the figure we see that the EMIC conics occur primarily in the pre-midnight sector. The figure shows that the EMIC conics are most common in the pre-midnight sector and rarely found near dawn; this distribution is consistent with the magnetic local time distribution that has been observed for EMIC waves alone (Saito et al., 1987; Erlandson and Zanetti, 1998).

In the auroral acceleration region ion cyclotron (e.g. Mozer et al., 1977; Ergun et al., 1998; Cattell et al., 1998a) and ion acoustic waves (e.g. Cattelle et al., 1998b) are sug-

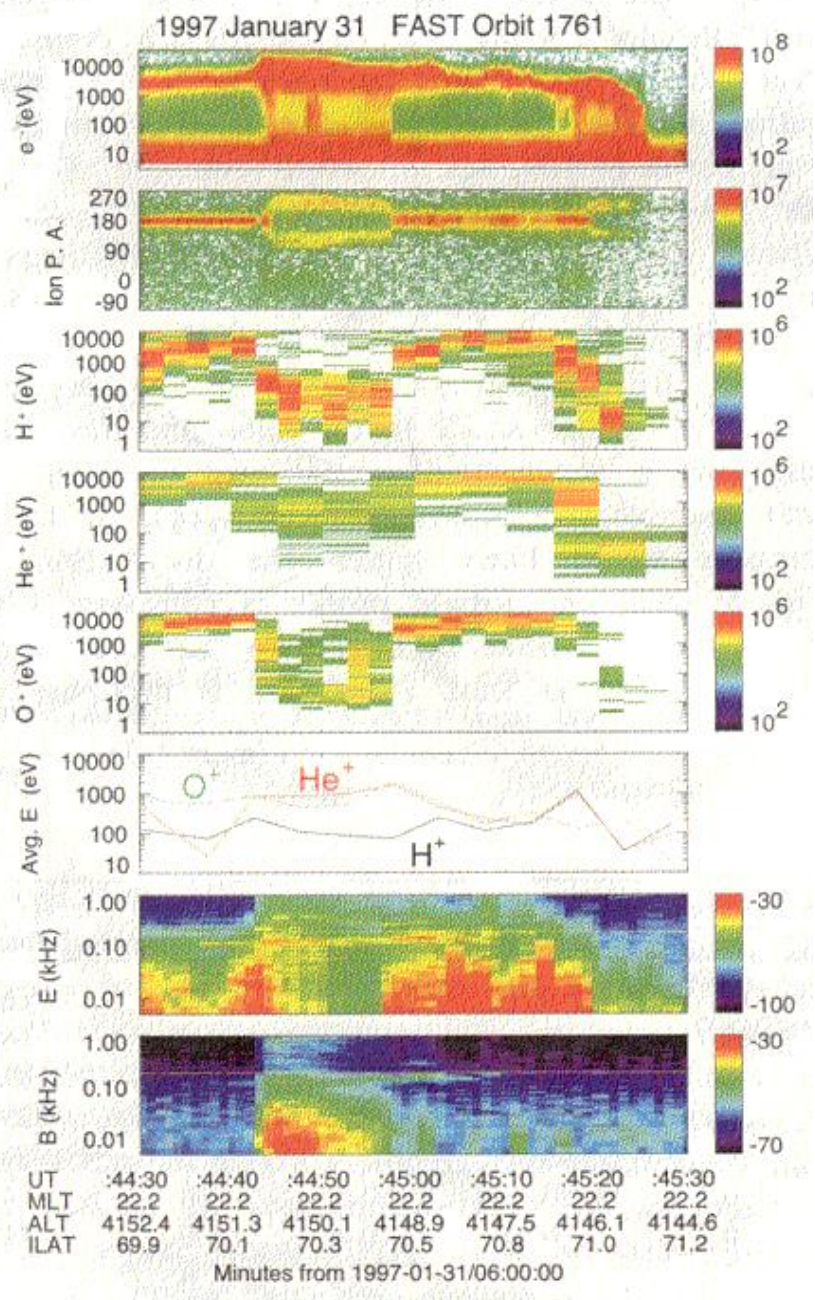

Fig. 1. Overview of the EMIC ion conics event from the nightside on orbit 1971 (Lund et al., 2000).

gested as possible modes. The parallel electric field signature (Hull et al., 2003), shown by Fig. 3, has a peak amplitude of $350 \mathrm{mV} / \mathrm{m}$, which occurs at $\sim 04: 17: 07$ UT in the region characterized by a sharp gradient in the plasma density (Fig. 3e). The parallel electric field signature is nearly coincident with large-amplitude perpendicular fields, characterized by a peak amplitude of $400 \mathrm{mV} / \mathrm{m}$ (Figs. 3c-d). The decreasing value of $\Delta B_{\gamma}$ (Fig. 3f) with increasing invariant latitude suggests that the event is characterized by an upward field-aligned current $\left(J_{\|}=0.17 \mu \mathrm{A} \mathrm{M}^{-2}\right)$. Upward field-aligned ion beams characterize the regions of depressed density, with peak energies reaching 5 to $6 \mathrm{keV}$ (Figs. 3h-i) with precipitating electrons (Figs. $3 \mathrm{j}-\mathrm{k}$ ). The variations in the plasma potential (Fig. 3g) are in rough accord with the beam energies of the ions (Hull et al., 2003).

Electrons streaming down magnetic field lines generate electromagnetic ion cyclotron (EMIC) waves just below the proton gyrofrequency, where they resonant with mirroring 


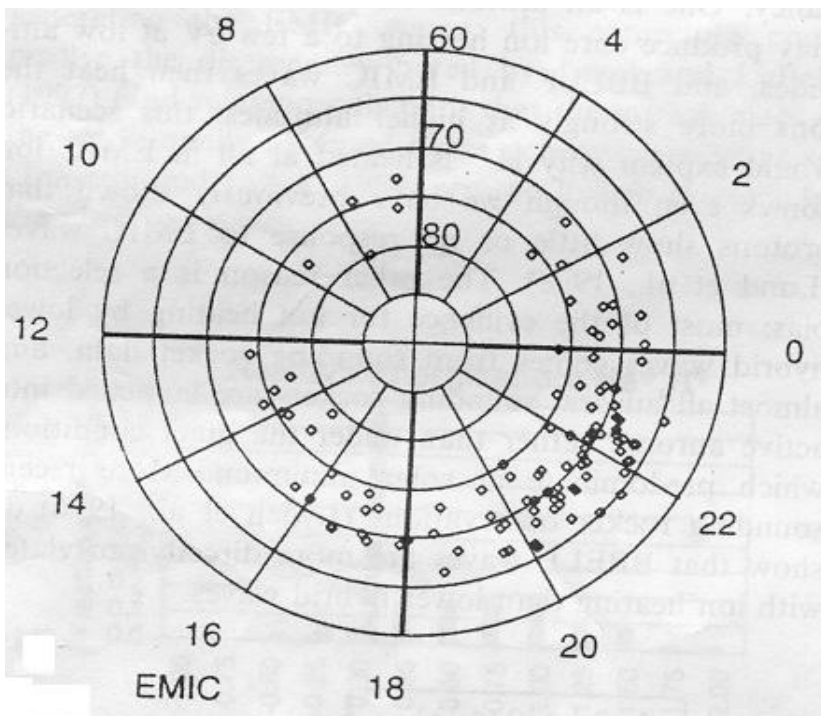

Fig. 2. Distribution of ion conic events over invariant latitude and magnetic local time: EMIC events (Lund et al., 2000).

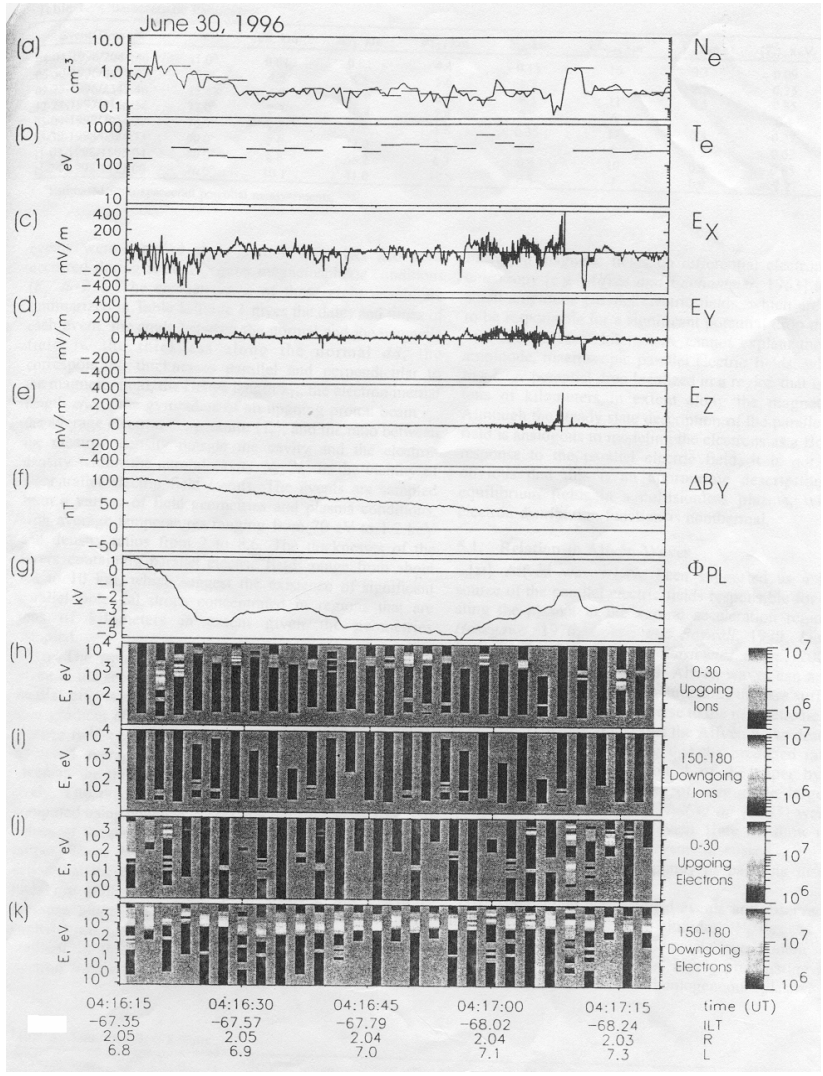

Fig. 3. Southern auroral zone crossing near dusk on 30 June 1996 (Hull et al., 2003).
${ }^{3} \mathrm{H}_{e}$ ions (Temerin and Roth, 1992; Roth and Temerin, 1997). A similar process produces the phenomena of "ion conics" in the Earth's aurora where the downward electron beams, EMIC waves and ions can all be observed in situ.

Based on the observational evidences (Lund et al., 2000, 2001; Jordonava et al., 2001; Chaston et al., 2002a, b; Hull et al., 2003) and theoretical model of particle aspect analysis (Misra and Tiwari, 1979; Tiwari and Varma, 1991; Bajaj and Tiwari, 1992; Varma and Tiwari, 1991, 1993; Dwivedi et al., 2001a, b, 2002; Mishra and Tiwari, 2006), the treatment of the EMIC instability in the ionosphere and magnetosphere may be important. It would be an essential contribution to the knowledge of the auroral ionosphere and to the plasma physicist in general.

\section{Mathematical considerations}

\subsection{Basic trajectory}

The present work is based upon the particle aspect analysis as adopted by Terashima (1967), Misra and Tiwari (1979), Tiwari and Varma (1991), Dwivedi et al. (2001a, b) and Mishra and Tiwari (2006) for the analysis of waves and instabilities. The detailed treatment of the EMIC wave, using particle aspect analysis, has been presented by Misra and Tiwari (1979). Following their approach the perturbed velocities of ions in the presence of an EMIC wave is given as

$$
\begin{aligned}
\delta V_{\perp} & =\frac{h \Omega\left(V_{\left.\Pi 0^{-}-\frac{\omega}{k}\right)}\right.}{k V_{\amalg^{0}}-(\omega-\Omega)}[\cos (k z-\omega t-\psi) \\
& \left.-\varepsilon \cos \left(k z-\omega t-\psi-\left(k V_{\prod_{0}}-(\omega-\Omega)\right) t\right)\right] \\
\delta V_{\Pi} & =\frac{-h V_{\perp 0} \Omega}{k V_{\amalg^{0}}-(\omega-\Omega)}[\cos (k z-\omega t-\psi \\
& \left.\left.-\varepsilon \cos \left(k z-\omega t-\psi-\left(k V_{\prod_{0}}-(\omega-\Omega)\right)\right) t\right)\right],
\end{aligned}
$$

where $\mathrm{z}=\mathrm{z}_{0}+\mathrm{V}_{\|} \mathrm{t}$ and $\psi=\psi_{0}-\omega \mathrm{t}$ and $\varepsilon=0,1$ for the nonresonant and resonant particles, $h=\frac{\boldsymbol{B}}{B_{0}}$ is the ratio of electromagnetic to static magnetic field amplitude and $\Omega=\frac{q B_{0}}{m c}$ is the ion cyclotron frequency; $\omega$ is wave frequency and $\mathrm{k}$ is the wave vector. All other symbols have their usual meanings. The density perturbations in the presence of an EMIC wave is given as (Misra and Tiwari, 1979).

$n_{1}=\frac{h V_{\perp 0} \Omega k N(V)}{\left[k V_{\prod 0}-(\omega-\Omega)\right]^{2}}\left[\cos \chi-\varepsilon \cos \chi_{0}+\varepsilon t \Lambda \sin (\chi-\Lambda t)\right]$

where $\chi=\mathrm{kz}-\omega \mathrm{t}-\psi$ and $\Lambda=\left(\mathrm{kV}_{|| 0^{-}}(\omega-\Omega)\right)$.

\subsection{General distribution function}

To investigate the energies, dispersion relation and growth rate, we choose the generalized distribution function of the following form as (Misra and Tiwari, 1979; Varma and Tiwari, 1993)

$N(V)=N_{0} f_{\perp}\left(V_{\perp}\right) f_{\Pi}^{\left(V_{\Pi}\right)}$ 
or

$$
N(V)=\frac{N_{0} V_{\perp}^{2 J}}{\pi^{3 / 2} V_{T \perp}^{2(J+1)} j !} \exp \left[-\frac{V_{\perp}^{2}}{V_{T \perp}^{2}}-\frac{V^{2}}{V_{T \prod c i}^{2}}\right]
$$

and

$$
T_{\prod c i}=T_{\prod}\left[1-i \frac{e \bar{E}_{0} \bar{k}}{k^{2} T_{\prod i K}}\right] .
$$

In the past, the general procedures for determining the plasma distribution along auroral field lines subject to a parallel potential drop are given by various workers (e.g. Smith and Langmuir, 1926; Whipple, 1977; Chiu and Schulz, 1978), by applying a Liouville type of analysis. In this paper we adopt an alternative approach based upon the Boltzmann equation for one particle distribution function $f_{ \pm}(\mathrm{r}, \mathrm{v}, \mathrm{t})$ and take into account the influence of charged particle interactions by means of a self-consistent field, as discussed in detail by Pines and Schrieffer (1961) for solid-state plasma and adopted in various papers for the magnetospheric wave analysis. Pines and Schrieffer have considered the collisionless Boltzmann equation for the distribution function $f_{ \pm}(\mathrm{r}, \mathrm{v}, \mathrm{t})$ in the presence of an external electric field $\mathrm{E}_{0}$ as

$$
\frac{\partial f_{ \pm}}{\partial t}+\boldsymbol{v} \cdot \nabla f_{ \pm} \pm \frac{e}{m_{ \pm}} \boldsymbol{E} \cdot \nabla_{v} f_{ \pm}=0
$$

and solved for the perturbed distribution $f_{1_{ \pm}}$, satisfying the equation

$i\left(\omega-k . v \pm \frac{e E_{0}}{m_{ \pm}} \frac{\partial}{\partial v_{z}}\right) f_{1}= \pm e \frac{e E_{s}}{m_{ \pm}} . \nabla_{v} f_{0 \pm}$,

where $E_{=} E_{0}+E_{s}, E_{s}$ is the perturbed electric field and all other symbols have their usual meanings. They have stated that it is straightforward to solve the above equation and the result is equivalent to replacing the temperatures $T_{ \pm}$by complex temperatures,

$T_{ \pm}^{\prime}=T_{ \pm}\left[1 \mp \frac{i e E_{0} k}{k^{2} K T_{ \pm}}\right]$.

This procedure was further adopted by various workers (Misra et al., 1979; Varma and Tiwari, 1991; Mishra and Tiwari, 2006).

$\mathrm{J}=0,1,2$ is the distribution index, known as the steepness of the loss-cone, $V_{T \Pi}^{2}=\left(\frac{2 T}{m}\right)$ and $V_{T \perp}^{2}=(J+1)^{-1}\left(\frac{2 T_{\perp}}{m}\right)$. For $\mathrm{J}=0, \mathrm{~N}(\mathrm{~V})$ goes to the Maxwellian distribution function and for $\mathrm{J}=\infty$, it behaves like a Dirac delta function $\delta\left(\mathrm{V}_{\perp}\right.$ $\left.\mathrm{J}^{1 / 2} \mathrm{~V}_{T \perp}\right)$. Moreover, $\mathrm{N}(\mathrm{V})$ is peaked about $\mathrm{J}^{1 / 2} \mathrm{~V}_{T \perp}$ and has a half width of $\Delta \mathrm{V}_{\perp} \sim \mathrm{J}^{-1 / 2} \mathrm{~V}_{T \perp}$, where $k=\left(k_{\perp}^{2}+k_{\| \mid}^{2}\right)^{1 / 2}$ and the expression for $T_{\Pi c i}$ is originally derived by Pines and Schrieffer (1961), adopting the rigorous treatment of a kinetic approach for the collective behavior of solid-state plasma. They have arrived at the results where the parallel electric field $E_{0}$ is eliminated by adopting the expression for
$T_{\prod c i}$, as shown above. Their work also described the wave vector $k$ at an angle to the $E_{0}$ (Eq. (2.24) of Pines and Schrieffer, 1961). They have clearly mentioned that the sign of the effect depends upon both the charge of the particle and the angle between $E_{0}$ and $k$. In this description the parallel electric field $E_{0}$ is sufficiently weak, so that the drift velocity of the charged particles is much smaller than the phase velocity of the wave. $e \Psi / T_{e}<1$ and time scales are such that the relaxations are nearly Maxwellian and the runaway conditions of the electrons are excluded by the same reasons as discussed by Tiwari and Varma (1991, and reference therein). Here we follow the techniques of Pines and Schrienffer (1961) and Bers and Brueck (1968), where a change in the zerothorder distribution function is due to the result of a change in temperature parallel to the parallel electric field $E_{0}$. This method was further considered by Misra et al. (1979) for the investigation of whistler mode instability, Varma and Tiwari (1991) for the investigation of drift instability, Dwivedi et al. (2001b) for kinetic Alfvén wave study and Mishra and Tiwari (2006) for EIC instability. The existence of the parallel static electric field in the presence of an EMIC wave in the auroral acceleration region may be a matter of further debate.

\subsection{Dispersion relation}

The existence of the ion energy anisotropy has been established and the growth is possible only when $\frac{T_{\perp}}{T_{U}}>1$. Thus, we are interested in the behavior of those particles for which $\frac{T_{\perp}}{T}>1$.Then we consider the cold plasma dispersion relation for the EMIC wave as (Misra and Tiwari, 1979)

$\frac{c^{2} k^{2}}{\omega^{2}}=\left(\frac{\omega_{p i}^{2}}{\Omega^{2}}\right)\left(1-\frac{\omega}{\Omega}\right)^{-1}$,

where $\omega_{p i, e}^{2}=\frac{4 \pi N_{0} e^{2}}{m_{i, e}}$ is the plasma frequency for the ions.

In the present analysis the effect of the parallel electric field has been introduced through the modification of temperature parallel to the magnetic field. In the cold plasma, the parallel electric field may not exist due to large conductivity along the magnetic field line. Thus, the cold plasma dispersion relation, which is independent of temperature, may be considered in the further analysis to evaluate the growth/damping rate.

\subsection{Wave energy and growth rate}

The wave energy density $\mathrm{W}_{w}$ per unit wavelength is the sum of the pure field energy and the changes in the energy of the non-resonant particles, i.e. the total energy per unit wavelength is given as

$W_{w}=U+W i$, 
where $U$ is the energy of electromagnetic wave as defined by the expression (Misra and Tiwari, 1979)

$U=\left(\frac{1}{16 \pi}\right)\left[\left(\frac{d}{d \omega}\right)\left(\omega \varepsilon_{i k}\right) E_{1}^{*} E_{k}+|B|^{2}\right]$,

where here $\varepsilon_{i k}$ is the dielectric tensor. After the calculation, the electromagnetic wave energy per unit wavelength is given by

$U=\left(\frac{\lambda B^{2}}{8 \pi}\right)\left[\frac{(2 \Omega-\omega)}{(\Omega-\omega)}\right]$.

Using the expressions for perturbed velocity and perturbed density and $\varepsilon=0$, the change in parallel non-resonance particles energy is given as (Misra and Tiwari, 1979)

$$
W_{i \prod}=\frac{-\lambda B^{2}}{8 \pi} \frac{C_{j}}{V_{T \prod c i}^{2}} \frac{\omega_{p i}^{2}}{c^{2} k^{2}}\left[\frac{1}{2} Z_{1}(\zeta)+\frac{\omega-\Omega}{k V_{T \prod c i}} Z_{2}(\zeta)\right]
$$

and perpendicular non-resonant energy as:

$$
\begin{aligned}
W_{i \perp} & =\frac{\lambda}{2} \frac{B^{2}}{8 \pi} \frac{\omega_{p i}^{2}}{c^{2} k^{2}}\left[D_{j}\left(1-\frac{2 \Omega}{k V_{T} \Pi c i} Z(\zeta)+\frac{\Omega^{2}}{k^{2} V_{T \Pi c i}^{2}} Z_{1}(\zeta)\right)\right. \\
& \left.+\frac{2 C_{j}}{V_{T \Pi c i}^{2}}\left(Z_{1}(\zeta)-\frac{\Omega}{k V_{T} \Pi c i} Z_{1}(\zeta)\right)\right] .
\end{aligned}
$$

The perpendicular (transverse) energy and the parallel resonant energy of the resonant ions are calculated with $\varepsilon=1$ as

$$
W_{r \perp}=\frac{\lambda}{16 \pi} \frac{B^{2} \omega_{p i}^{2}}{C^{2} k^{2}} t\left[C_{J}+\frac{2 \Omega^{2}}{k^{2}} D_{J}\right] f_{r}\left(V_{r}\right)-C_{J} \frac{\Omega}{k} f^{\prime}\left(V_{r}\right)(
$$

and

$$
W_{r \amalg}=\frac{\lambda}{16 \pi} \frac{B^{2} \omega_{p i}^{2}}{c^{2} k^{2}} t C_{J}\left(\frac{(\omega-\Omega)}{k}\right) f^{\prime}\left(V_{r}\right),
$$

where

$$
\begin{aligned}
& C_{J}=\frac{1}{V_{T \perp}^{2(J+1)} J !} \int_{0}^{\infty} d V_{\perp}^{2} V_{\perp}^{2(J+1)} \exp \left(-\frac{V_{\perp}^{2}}{V_{T \perp}^{2}}\right) \\
& D_{J}=\frac{1}{V_{T \perp}^{2(J+1)} J !} \int_{0}^{\infty} d V_{\perp}^{2} V_{\perp}^{2 J} \exp \left(-\frac{V_{\perp}^{2}}{V_{T \perp}^{2}}\right)
\end{aligned}
$$

and

$$
\begin{aligned}
& f_{r}\left(V_{r}\right)=\left(\frac{m}{2 \pi T_{\prod c i}}\right)^{1 / 2} \exp \left[-\frac{m(\omega-\Omega)^{2}}{2 T_{\prod c i} k^{2}}\right] \\
& f^{\prime}\left(V_{r}\right)=-2\left(\frac{m}{2 \pi T_{\prod c i}}\right)^{1 / 2}\left(\frac{\omega-\Omega}{k V_{T \prod c i}^{2}}\right) \exp \left[-\left(\frac{\omega-\Omega}{k V_{T \prod c i}}\right)^{2}\right] .
\end{aligned}
$$

Using the law of conservation of energy. The growth/damping rate $\gamma$ is derived as (Misra and Tiwari, 1979)

$$
\frac{\gamma}{\omega}=\frac{\pi^{1 / 2} \frac{\Omega^{2}}{k^{2} V_{T \prod i}^{2} S}\left[\frac{(\Omega-\omega)}{\Omega} \frac{(J+1) T_{\perp}}{T_{\amalg i} S}-1\right] \exp \left[-\frac{1}{V_{T}^{2} \amalg i}\left(\frac{\omega-\Omega}{k}\right)^{2}\right]}{\left(\frac{c k}{\omega_{p i}}\right)^{2}\left(\frac{2 \Omega-\omega}{\Omega-\omega}\right)+\frac{1}{2} \frac{\omega^{2}}{(\Omega-\omega)^{2}}},
$$

where $S=\left[1+\frac{e^{2} E_{0}^{2}}{k^{2}\left(k T_{\Pi i)^{2}}\right.}\right]$.

Here it is noticed that the parallel electric field $\mathrm{E}_{0}$ has affected the growth rate and change in the energy for the electromagnetic waves propagating parallel to the magnetic field with general loss-cone distribution function.

\subsection{Marginal instability}

For the marginal instability condition $\gamma=0$, the maximum stable frequency is obtained

$$
\omega=\Omega\left[1-\frac{1}{(J+1)} \frac{T_{\perp} S}{T_{\perp}}\right] .
$$

\section{Result and discussion}

The magnetosphere-ionosphere coupling has the ability in a plasma to support the magnetic field-aligned (parallel) electric potential drops, as indicated by various observations. The Earth's magnetic field electrically and dynamically decouples the region. Electric fields existing along geomagnetic field lines in the magnetospheric region play a significant role in the wave particle interaction. The computational analysis of resonant and non-resonant energy by Eqs. (12, 13) and growth rate by Eq. (14) are shown in Figs. 4 to 12. In the numerical calculation of the growth rate and changes in energies of the particles in the presence of the EMIC wave, we have used the following parameters of the auroral acceleration region (Tiwari and Rostoker, 1984; Hull et al., 2003); $\mathrm{B}_{0}=4300 \mathrm{nT} ; \Omega_{i}=412 \mathrm{~s}^{-1} ; \mathrm{V}_{T \|}=6.41 \times 10^{8} \mathrm{~cm} / \mathrm{s}$; $\omega_{p i}^{2}=1.732 \times 10^{6} \mathrm{~s}^{-2}$.

Figures 4-6 predict the variation of the normalized growth rate $\gamma / \Omega_{i}$ with $\mathrm{k}_{\|}\left(\mathrm{cm}^{-1}\right)$ at different values of the parallel electric field $\mathrm{E}_{0}$ for $\mathrm{J}=0,1,2$, respectively. It is noticed that the effect of the electric field is to reduce the growth rate of the EMIC wave. Thus, the parallel electric field controls the wave amplification in the magnetosphere and transfers the energy into the particle acceleration. It is also noticed by these figures that at higher values of $\mathrm{J}$, the growth rate is enhanced. The steep loss cone structures are analogous to mirror-like devices with a higher mirror ratio that may generate EMIC waves. Thus, energetic particles available may provide energy to the wave through wave particle interaction. Hence, the mirror-like structures of the magnetosphere with a steep loss cone distribution may be unstable for the EMIC wave emission.

The growth rate versus parallel wave number (Figs. 4-6) shows a monotonic increase in the linear limit. However, we obtain the growth rate at higher wave numbers $k_{U}$ but it has increased the ion cyclotron frequency, which violates the condition $\frac{\gamma}{\Omega_{i}}<1$. Under this condition a nonlinear treatment may be considered in our theoretical limit. In the subsequent perpendicular and parallel energy calculations this condition was also considered. 


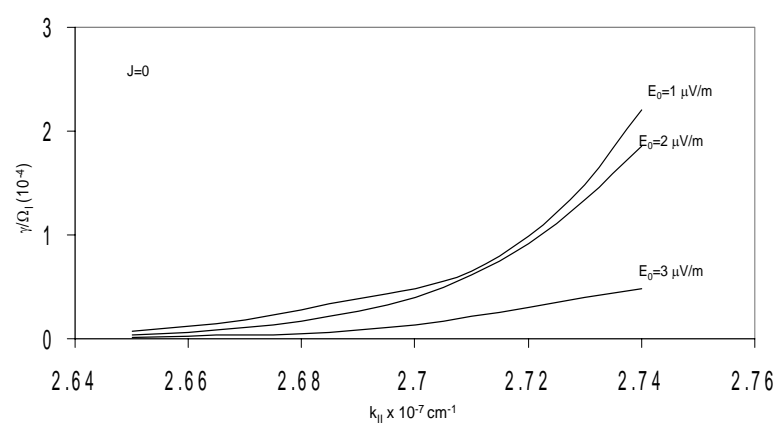

Fig. 4. Variation of growth rate $\left(\gamma / \Omega_{i}\right)$ versus wave vector $\mathrm{k}_{I I}$ $\mathrm{cm}^{-1}$ for different values of parallel electric field $\mathrm{E}_{0}$, and distribution index $\mathrm{J}=0$.

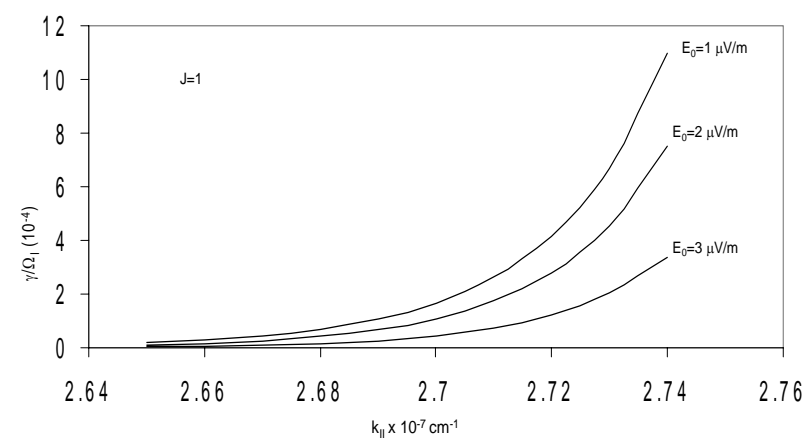

Fig. 5. Variation of growth rate $\left(\gamma / \Omega_{i}\right)$ versus wave vector $\mathrm{k}_{I I}$ $\mathrm{cm}^{-1}$ for different values of parallel electric field $\mathrm{E}_{0}$, and distribution index $\mathrm{J}=1$.

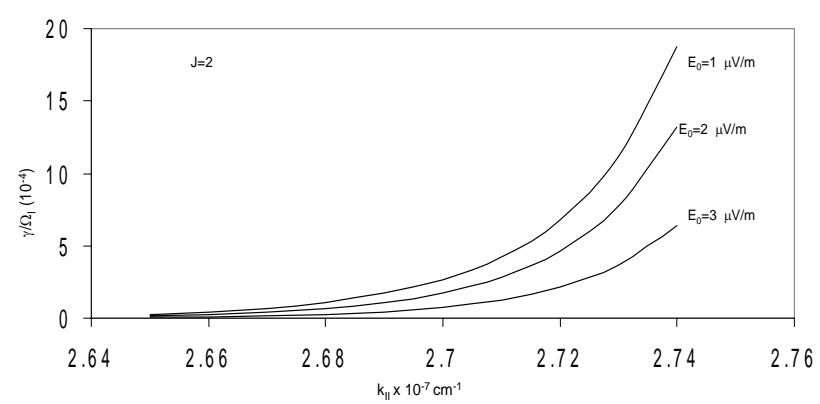

Fig. 6. Variation of growth rate $\left(\gamma / \Omega_{i}\right)$ versus wave vector $\mathrm{k}_{I I}$ $\mathrm{cm}^{-1}$ for different values of parallel electric field $\mathrm{E}_{0}$, and distribution index $\mathrm{J}=2$.

Figures 7-9 show the variation of the transverse resonant energy per unit wavelength $\left(\mathrm{W}_{r \perp}\right)$ in erg $\mathrm{cm}$, with $\mathrm{k}_{\|}\left(\mathrm{cm}^{-1}\right)$ at different values of the parallel electric field $\left(\mathrm{E}_{0}\right)$ and $\mathrm{J}=0$, 1,2 , respectively. It is observed that the effect of increasing the values of the parallel electric field $\left(\mathrm{E}_{0}\right)$ is to enhance the transverse resonant energy of the ions. The correlation of the EMIC ion conics with phenomena which are associated with parallel electric fields play a significant role in transverse ion

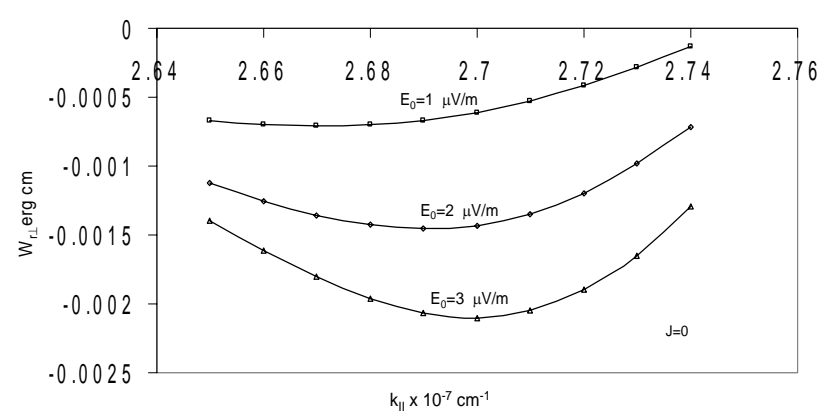

Fig. 7. Variation of perpendicular resonant energy $\mathrm{Wr}_{\perp} \mathrm{erg} \mathrm{cm}$ versus wave vector $\mathrm{k}_{I I} \mathrm{~cm}^{-1}$ for different values of parallel electric field $\mathrm{E}_{0}$, and distribution index $\mathrm{J}=0$.

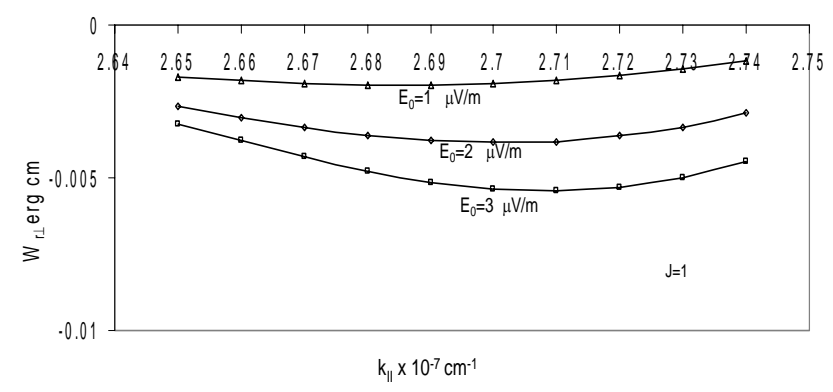

Fig. 8. Variation of perpendicular resonant energy $\mathrm{Wr}_{\perp}$ erg cm versus wave vector $\mathrm{k}_{I I} \mathrm{~cm}^{-1}$ for different values of parallel electric field $\mathrm{E}_{0}$, and distribution index $\mathrm{J}=1$.

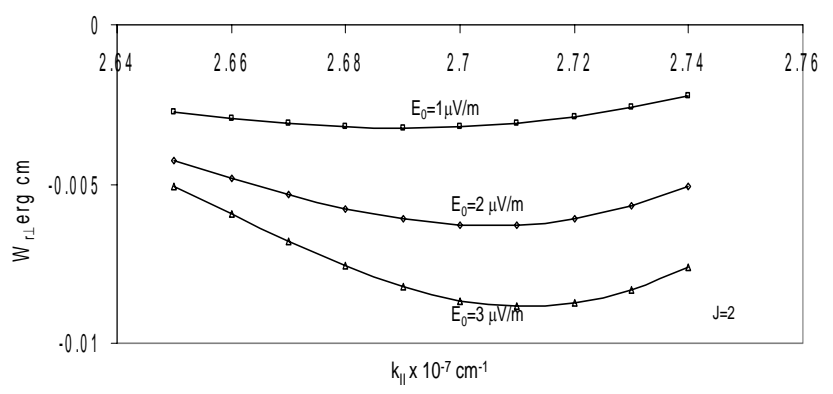

Fig. 9. Variation of perpendicular resonant energy $\mathrm{Wr}_{\perp}$ erg cm versus wave vector $\mathrm{k}_{I I} \mathrm{~cm}^{-1}$ for different values of parallel electric field $\mathrm{E}_{0}$, and distribution index $\mathrm{J}=2$.

heating in the aurora; this is in accordance with the observations as reported by Lund et al. (2000). The particles having a greater velocity as compared to the phase velocity of the wave provide energy to the wave via cyclotron interaction and the wave growth is possible. The increase in heating of the resonant ions by the parallel electric field is supported by the decrease in the growth rate, as the EMIC wave energy is being transferred to the ions by the resonance interaction 


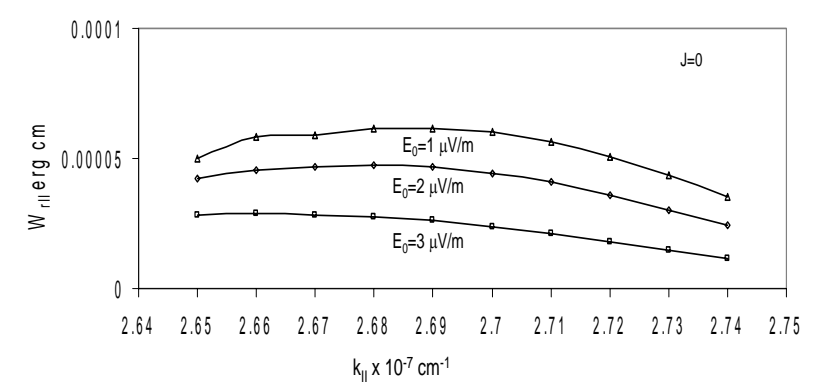

Fig. 10. Variation of parallel resonant energy $\mathrm{Wr}_{I I} \mathrm{erg} \mathrm{cm}$ versus wave vector $\mathrm{k}_{I I} \mathrm{~cm}^{-1}$ for different values of parallel electric field $\mathrm{E}_{0}$, and distribution index $\mathrm{J}=0$.

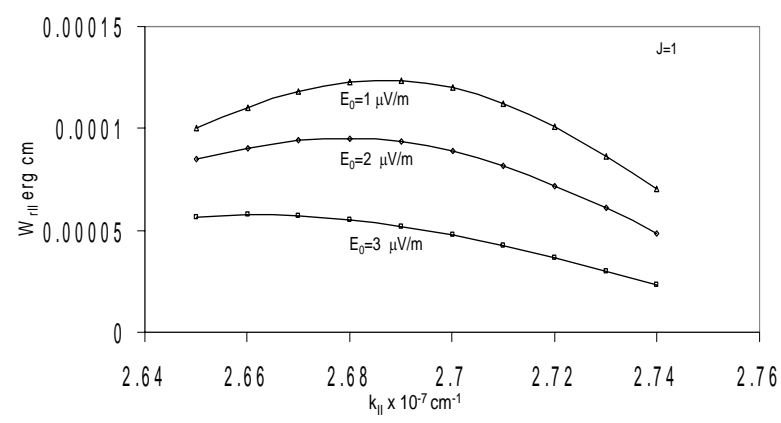

Fig. 11. Variation of parallel resonant energy $\mathrm{Wr}_{I I} \mathrm{erg} \mathrm{cm}$ versus wave vector $\mathrm{k}_{I I} \mathrm{~cm}^{-1}$ for different values of parallel electric field $\mathrm{E}_{0}$, and distribution index $\mathrm{J}=1$.

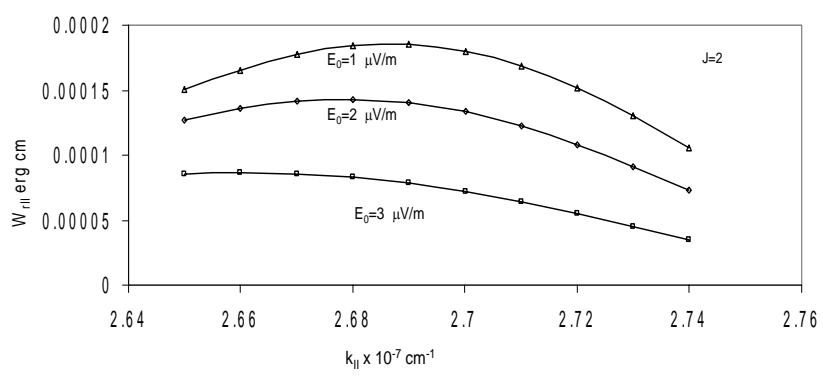

Fig. 12. Variation of parallel resonant energy $\mathrm{Wr}_{I I} \mathrm{erg} \mathrm{cm}$ versus wave vector $\mathrm{k}_{I I} \mathrm{~cm}^{-1}$ for different values of parallel electric field $\mathrm{E}_{0}$, and distribution index $\mathrm{J}=2$.

process. The effect of increasing the values of the distribution index $\mathrm{J}=0,1,2$, is to decrease the transverse resonant energy of the ions as the growth rate is enhanced. Thus, the steepness of the loss-cone distribution decreases the transverse resonant energy of the ions in the presence of EMIC waves.

Figures 10-12 predict the relation between the parallel resonant energy per unit wavelength of the ions $\left(\mathrm{W}_{r \|}\right)$ in erg $\mathrm{cm}$, and $\mathrm{k}_{\|}\left(\mathrm{cm}^{-1}\right)$ at different values of the parallel electric field $E_{0}$ at $J=0,1,2$, respectively. The effect of increasing values of the parallel electric field $\mathrm{E}_{0}$ is to decrease the parallel resonant energy due to the EMIC wave, which causes the increase in the transverse resonant energy of the ions. The effect of increasing the values of the distribution index $\mathrm{J}$ is to show the increasing effect of the parallel resonant energy of the ions. Thus, the steepness of the loss-cone distribution is to increase both the energies $\mathrm{W}_{r \perp}$ and $\mathrm{W}_{r \| \mid}$ in the presence of an EMIC wave.

The effect of the loss-cone distribution is to enhance the growth rate of the EMIC wave, as well as to increase the energies of the resonant ions in the presence of the wave. The transversely accelerated ions and their association with EMIC waves in the auroral acceleration region have been recently reported by various workers (Lund et al., 1999, 2000, 2001; Chaston et al., 2002a,b) in the analysis of the FAST satellite data (Strangeway et al., 1998). The EMIC ion conics and related phenomena are associated with parallel electric fields, suggesting that parallel electric fields play a significant role in transverse ion heating in the aurora. The parallel electric fields and steep loss-cone distributions both enhance the perpendicular energy of the ions in the auroral acceleration region. The equilibrium dipolar magnetic field of the Earth is curved in a meridonial plane and introduces the losscone effects in the particle distribution function (Varma and Tiwari, 1992; Tiwari and Varma, 1991, 1993; Dwivedi et al., 2001b). Hence, the study of the EMIC wave in the auroral acceleration region with the parallel electric field and steep loss-cone distribution is of importance to explain the observations of the FAST satellite (Lund et al., 2000, 2001).

Measurement of the parallel electric field is a recent aspect of rocket and satellite experiments in space plasma (Mozer and Hull, 2001; Hull et al., 2003). Theoretical and experimental studies have indicated the presence of parallel electric fields in the range from several microvolts per meter to several mill volts per meter. The electric field observed along the auroral field lines in the presence of EMIC wave may govern the behavior of auroral electrodynamics. The electrical energy may be transferred to the ions by the EMIC wave. The EMIC waves may be excited in the auroral acceleration regions, as predicted by the enhancement of growth rates with various parameters, but the role of the observed electric field is to control the growth rate in the linear limit.

Coulomb collisions between energetic ring current ions and thermal plasma result in energy transfer from the fast moving particles to the thermal particles and in angular deflection of the particles (Wentworth et al., 1959). The magnetospheric energy is transported down the magnetic field lines and creates ionospheric electron and ion temperature enhancements (Brace et al., 1967) and optical emissions known as stable auroral red (SAR) arcs (Cole, 1965). The pitch angle diffusion caused by Columb collisions scatters particles into the loss cone and contributes to the increase of the precipitating ion flux at low altitudes.

Ring current ion distributions are often unstable and can generate different classes of plasma waves in the equatorial 
magnetosphere, such as magnetosonic, electromagnetic ion cyclotron (EMIC) waves, etc. The subsequent interaction of energetic particles with the plasma wave results in pitch angle scattering and energy exchange between the particle and the wave. Scattering of ring current particles into the loss cone, due to resonant interactions with EMIC waves, occurs on short time scales and could contribute significantly to ion loss (Cornwall et al., 1970; Lyons and Thorne, 1972), especially during the main phase of the storm, when ring current energy loss time scales may be as low as $0.5-1 \mathrm{~h}$ (Gonzalez et al., 1989). The plasma waves can also transfer energy from ring current $\mathrm{H}^{+}$to $\mathrm{O}^{+}$during magnetic storms (Thorne and Horne, 1994, 1997) and play an important role in the heating of the thermal electrons and ions (Horne and Thorne, 1997).

In the present analysis, we have considered only one ion species. However, in the auroral acceleration region, there are several ion species drifting relative to each other. The presence of various drifting ion species affects the cold plasma dispersion relation and growth rate in substantial way (Gomberoff and Neira, 1983; Gomberoff and Elgueta, 1991; Gomberoff et al., 1996). These effects cannot be neglected and may be the matter of further investigation. However, the present basic model can be useful to explain some of the observations qualitatively and may be useful to explain perpendicular energisation in the extended solar corona (Cranmer and Ballegooijen, 2003).

In the present analysis we have adopted the particle aspect analysis and have arrived at the result reported by Gomberoff and Cuperman (1981), using a kinetic approach, where temperature anisotropy $\frac{T_{\perp}}{T_{U}}$ is replaced by effective temperature anisotropy $(\mathrm{J}+1) \frac{T_{\perp}}{T_{\amalg}}$. We recover all the results of the kinetic approach by using a generalized loss-cone distribution, making the above replacement of the Maxwellian distribution for EMIC waves propagating along the magnetic field (Summers and Thorne, 1995).

The particle aspect analysis developed in the present paper may offer its relative advantages by considering the energy transfer between wave and particles perpendicular and parallel to the magnetic field, along with a discussion of wave emission in auroral acceleration region. The results obtained using this approach confirms the general results of Gomberoff and Cupermam (1981). The main objective of the present investigation is to examine the effect of the parallel electric field on EMIC waves at different loss-cone indices J, in view of the observations in the auroral acceleration region.

It is widely recognized that the electric field plays an important role in the dynamics of the plasma in the ionosphere, as well as in the magnetosphere and the solar wind. In the auroral regions charged particles are accelerated to very high energies by the electric field parallel to the magnetic field. In the auroral zone a quasi-static parallel electric field accelerates ionospheric ions outward, providing a significant source of ions for the magnetosphere. Such an electric field acceler- ates the ions to approximately the same energy, irrespective of their mass. The presence of the parallel electric field and the EMIC wave in the acceleration region may control the heating rate of plasma particles along and perpendicular to the magnetic field. The particle aspect analysis developed may be applicable to laboratory plasma, as well as to estimate the heating rates, along with the study of emissions of EMIC waves.

Acknowledgements. P. Varma is thankful to Department of Science $\&$ Technology (DST), New Delhi for financial assistance.

Topical Editor I. A. Daglis thanks L. Gomberoff and another referee for their help in evaluating this paper.

\section{References}

Andre, M., Norqvist, P., Andersson, L., Eliasson, L., Eriksson, A. I., Blomberg, L., Erlandson, R. E., and Waidemark, J.: Ion energization mechanisms of $1700 \mathrm{~km}$ in the auroral region, J. Geophys. Res., 103, 4199-4222, 1998.

Arnoldy, R. L., Moore, T. E., and Cahill, L. J.: Low-amplitude field aligned Electrons, J. Geophys. Res., 90, 8445-8451, 1985.

Bajaj, V. K. and Tiwari, M. S.: Effect of parallel electric field on the electrostatic ion cyclotron instability, Ind. J. Radio \& Space Phys., 21, 53-63, 1992.

Bers, A. and Brueck, S. R.: Acoustic wave amplification at microwave frequency, Quart. Progr. Res. Rep., 89, 156, 1968.

Borovsky, J. E.: The strong-double-layer model of auroral arcs: An assessment in auroral plasma dynamics, Geophys. Monogr. Ser., edited by: Lysak, R. L., 80, AGU, Washington, D.C., 113-120, 1993.

Brace, L. H., Reddy, B. M., and Mayr, H. G.: Global behavior of the ionosphere of 1000 kilometer altitude, J. Geophys. Res., 72, 265-283, 1967.

Cattell, C. A., Wygant, J. R., Dombeck, J., and Mozer, F. S.: The association of electrostatic ion cyclotron waves, ion and electron beams and field-aligned currents: FAST observations of an auroral zone crossing near midnight, Geophys. Res. Lett., 25, 20532056, 1998a.

Cattell, C. A., Wygant, J. R., Dombeck, J., Mozer, F. S., Temerin, M. A., and Russell, C. T.: Observations of large-amplitude parallel electric field wave packets at the plasma sheet boundary, Geophys. Res. Lett., 25, 857-860, 1998b.

Chaston, C. C., Ergun, R. E., Delory, G. T., et al.: Characteristics of electromagnetic proton cyclotron waves along auroral field lines observed by FAST in region of upward current, Geophys. Res. Lett., 25, 2057-2064, 1998.

Chaston, C., Carlson C. W., Peria, W. J. Ergun, R. E., and McFadden, J. P.: FAST observations of inertial Alfven waves in the dayside aurora, Geophys. Res. Lett., 26, 647-650, 1999.

Chaston, C. C., Hu, Y. D., and Fraser, B. J.: Quasi-linear ion cyclotron heating in the near Earth magnetotail, J. Geophys. Res., 105, 5507-5516, 2000.

Chaston, C. C., Bonnell, J. W., Peticolas, L. M., Carlson, C. W., McFadden, J. P., and Ergun, R. E.: Driven Alfven waves and electron acceleration: A FAST case study, Geophys. Res. Lett., 29(11), 1535, doi:10.1029/2001GLO13842, 2002a.

Chaston, C. C., Bonnell, J. W., Carlson, C. W., Berthomier, M., Peticolas, L. M., Roth, I., McFadden, J .P., Ergun, R. 
E., and Strangeway, R. J.: Electron acceleration in the ionospheric Alfven resonator, J. Geophys. Res., 107, (A11), 1413, doi:10.1029/2002JA009272, 2002b.

Chiu, Y. T. and Schulz, M.: Self consistent particle and parallel electrostastic field distributions in the magnetosphere-ionosphere auroral region, J. Geophys. Res., 83, 629-642, 1978.

Cole, K. D.: Stable auroral red arcs, sinks for energy of Dst main phase. J. Geophys. Res., 70, 1689-1706, 1965.

Cornwall, J. M., Coroniti, F. V., and Thorne, R. M.: Turbulent loss of ring current protons, J. Geophys. Res., 75, 4699-4709, 1970.

Cranmer, S. R. and van Ballegooijen, A. A.: Alfvenic turbulence in the extended solar corona: kinetic effects and proton heating, Astrophys. J., 594, 573-591, 2003.

Duan, S. P., Li, Z. Y., and Liu, Z. X.: Kinetic Alfven wave driven by the density inhomogeneity in the presence of loss-cone distribution function-particle aspect analysis, Planet. Space Sci., 53, 1167-1173, 2005.

Dwivedi, A. K., Varma, P., and Tiwari, M. S.: Kinetic Alfven wave in the inhomogeneous magnetosphere and general distribution function, Planet. Space Sci., 49, 993-1003, 2001a.

Dwivedi, A. K., Varma, P., and Tiwari, M. S.: Kinetic Alfven wave in the presence of parallel electric field in an inhomogeneous magnetosphere, Ind. J. Phys., 75B(5), 555-563, $2001 \mathrm{~b}$.

Dwivedi, A. K., Varma, P., and Tiwari, M. S.: Ion and electron beam effects on kinetic Alfven waves in an inhomogeneous magnetosphere, Planet. Space Sci., 50, 93-99, 2002.

Erlandson, R. E. and Anderson, B. J.: Pc1 waves in the ionosphere: A statistical study, J. Geophys. Res., 101, 7843-7857, 1996.

Erlandson, R. E. and Zanetti, L. J.: A statistical study of auroral electromagnetic ion cyclotron waves, J. Geophys. Res., 103, 4627-4636, 1998.

Ergun, R. E., Carlson, C. W., McFadden, J. P., et al.: FAST satellite observations of electric field structures in the auroral zone, Geophys. Res. Lett., 25, 2025-2028, 1998a.

Evans, D. S.: The observation of near monoenergetic flux of auroral electron, J. Geophys. Res., 73, 2315-2323, 1968.

Evans, D. S.: Precipitation electron fluxes formed by a magnetic field aligned potential difference, J. Geophys. Res., 79, 28532858, 1974.

Genot, V., Lonam, P., and LeQnean, D.: A study of the propagation of Alfven waves in the auroral density acoustics, J. Geophys. Res., 104, 22 649-22 656, 1999.

Goertz, C. K. and Boswell, R. W.: Magnetosphere-ionosphere coupling, J. Geophys. Res., 84, 7239-7246, 1979.

Gomberoff, L. and Cuperman, S.: On the kinetic instabilities of uniform magnetized plasmas with general loss-cone distribution functions, J. Plasma Phys., 25, 99-102, 1981.

Gomberoff, L. and Neira, R.: Convective growth rate of ion cyclotron waves in a $\mathrm{H}^{+}-\mathrm{He}^{+}$and $\mathrm{H}^{+}-\mathrm{He}^{+}-\mathrm{O}^{+}$plasma, J. Geophys. Res., 88, 2170-2174, 1983.

Gomberoff, L. and Elgueta, R.: Resonant acceleration of alpha particles by ion cyclotron waves in the solar wind, J. Geophys. Res., 96, 9801-9804, 1991.

Gomberoff, L., Gnavi, G., and Gratton, F. T.: Minor heavy electromagnetic beam - plasma interactions in the solar wind, J. Geophys. Res., 101, 13 517-13 522, 1996.

Gonzalez, W. D., Tsuruatani, B. T., Gonzalez, A. L. C., Smith, E. J., Tang, F., and Akasofu, S.-I.: Solar wind magnetosphere coupling during intense magnetic storms, J. Geophys. Res., 94,
8835-8851, 1989.

Gorney, D. J., Clarke, A., Croley, D., Fennell, J., Luhnmann, J., and Mizera, P.: The distribution of ion beams and conics below 8000 km., J. Geophys. Res., 86, 83-89, 1981.

Gurnett, D. A. and Frank, L. A.: Observed relationships between electric field and auroral particle precipitation, J. Geophys. Res., 78, 145-170, 1973.

Horne, R. B. and Thorne, R. M.: Wave heating of $\mathrm{H}_{e}^{+}$by electromagnetic ion cyclotron waves in the outer magnetosphere: Heating near the $\mathrm{H}^{+}-\mathrm{He}^{+}$bi-ion resonance frequency, J. Geophys. Res.,102, 11 457-11471, 1997.

Hudson, M. K.: Magnetic field-aligned potential drops due to electrostatic ion cyclotron Turbulence, Geophys. Res. Lett., 5, 143146, 1978.

Hull, A. J., Bonnell, J. W., Mozer, F. S., and Scudder, J. D.: A statistical study of large-amplitude parallel electric field in the upward current region of the auroral acceleration region, J. Geophys. Res., 108(A1), 1007, doi:10.1029/2001JA007540, 2003.

Johnstone, A. D. and Winningham, J. D.: Satellite observations of suprathermal electron bursts, J. Geophys. Res., 87, 2321-2329, 1982.

Jordanova, V. K., Farrugia, C. J., Thorne, R. M., Khazanov, G. V., Reeves, G. D., and Thomsen, M. F.: Modeling ring current proton precipitation by electromagnetic ion cyclotron waves during the May 14-16, 1997 storm, J. Geophys. Res., 106, 7-21, 2001.

Kintner, P. M., LaBelle, J., Scales, W., Yau, A. W., and Whalen, B. A.: Observations of plasma waves within regions of perpendicular ion acceleration, Geophys. Res. Lett. 13, 1113-1116, 1986.

Kletzing, C. A.: Electron acceleration by kinetic Alfven waves, J. Geophys. Res., 99, 11 095-11 104, 1994.

Kletzing, C. A. and Hu, S.: Alfven wave generated electron time dispersion, Geophys. Res. Lett., 28, 693-696, 2001.

Klumpar, D. M., Peterson, W. K., and Shelley, E. G.: Direct evidence for two-stage (bimodal) acceleration of ionospheric ions, J. Geophys. Res., 89, 10 779-10 787, 1984.

Knudsen, D. J., Clemmons, J. H., and Wahlund, J. E.: Correlation between core ion energization, suprathermal electron bursts and broadband ELF plasma waves, J. Geophys. Res., 103, 41714186, 1998a.

Lund, E. J., Mobius, E., Klumpar, D. M., Kistler, L. M., Popecki, M. A., Klecker, B., Ergun, R. E., McFadden, J. P., Carlson, C. W., and Strangeway, R. J.: Occurrence distribution of preferentially heating events in the Aurora, Adv. Space Res., 23(10), 17211724, 1999.

Lund, E. J., Mobius, E., Carlson, C. W., Ergun, R. E., Kistler, L. M., Klecker, B., Klumpar, D. M., McFadden, J. P., Popecki, M. A., Strangeway, R. J., and Tung, Y. K.: Transverse ion acceleration mechanism in the aurora at solar minimum: Occurrence Distributions, J. Atmos. Solar-Terr. Phys., 62, 467-475, 2000.

Lund, E. J., Mobius, E., Lynch, K. A., Klumpar, D. M., Peterson, W. K., Ergun, R. E., and Carlson, C. W.: On the Mass dependence of transverse ion acceleration by broad- band extremely low frequency waves, Phys. Chem. Earth@, 26, 161-163, 2001.

Lyons, L. R. and Thorne, R. M.: Parasitic pitch angle diffusional of radiation belt particles by ion cyclotron waves, J. Geophys. Res., 77, 5608-5616, 1972.

Lynch, K. A., Arnoldy, R. L., Kintner, P. M., and Bonnell, J.: The AMICIST auroral sounding rocket: a comparison of transverse ion acceleration mechanisms, Geophys. Res. Lett , 23, 3293- 
3296, 1996.

Lysak, R. L. and Hudson, M. K.: Coherent anomalous resistvity in the region of electrostatic shocks, Geophys. Res. Lett., 6, 661$663,1979$.

Lysak, R. L. and Dum, C. T.: Dynamics of magnetosphereionosphere coupling including turbulent transport, J. Geophys. Res., 88, 365-380, 1983.

Marklund, G. T., Ivanchenko, N., and Karlsson, T.: Temporal evolution of acceleration structures in the auroral region, Nature, 414, 724-727, 2001.

McFadden, J. P., Carlson, C. W., Ergun, R. E., Mozer, F. S., et al.: Spatial structure and gradient of ion beams observed by FAST, Geophys. Res. Lett., 25, 2021-2024, 1998.

McFadden, J. P., Ergun, R. E., Elphic, R., Cattell, C. Pfaff, R., et al.: Electron modulation and ion cyclotron waves observed by FAST, Geophys. Res. Lett., 25, 2045-2048, 1998a.

McFadden, J. P., Carlson, C. W., and Ergun, R. E.: Microstructure of the auroral acceleration region as observed by FAST, J. Geophys. Res., 104, 14 453-14 480, 1999.

Misra, K. D. and Tiwari, M. S.: Particle aspect analysis of electromagnetic ion cyclotron Instability, Can. J. Phys., 57, 1124-1130, 1979.

Misra, K. D., Singh B. D., and Mishra, S. P.: Effect of parallel electric field on the whistler mode instability in the magnetosphere, J. Geophys. Res., 84, 5923-5928, 1979.

Mishra, R. and Tiwari, M. S.: Effect of parallel electric field on electrostatic ion cyclotron instability in anisotropic plasma in the presence of ion beam and general distribution function, Planet. Space Sci., 54, 188-199, 2006.

Miyake, W., Mukai, T., Kaya, N., and Fukunishi, H.: EXOS-D observations of upflowing ion conics with high time resolution, Geophys. Res. Lett., 18, 341-344, 1991.

Miyake, W., Mukai, T., and Kaya, N.: On the origins of the upward shift of elevated (bimodal) ion conics in velocity space, J. Geophys. Res., 101, 26 961-26 969, 1996.

Mozer, F. S. and Fahleson, U. V.: Parallel and perpendicular electric fields in an Aurora, Planet. Space Sci., 18, 1563-1571, 1970.

Mozer, F. S., Carlson, C. A., Hudson, M. H., et al.: Observations of paired electrostatic shocks in the polar magnetosphere, Phys. Rev. Lett., 38, 292-295, 1977.

Mozer, F. S., Cattell, C. A., Hudson, M. H., et al.: Satellite measurements and theories of low altitude auroral particle acceleration, Space Sci. Rev., 27, 155-213, 1980.

Mozer, F. S. and Kletzing, C. A.: Direct observation of large quasistatic parallel electric field in the auroral acceleration region, Geophys. Res. Lett., 25, 1629-1632, 1998.

Mozer, F. S. and Hull, A.: The origin and geometry of upward parallel electric field in the auroral acceleration region, J. Geophys. Res., 106, 5763-5778, 2001.

Papadopoulos, K. A.: Review of anomalous resistivity for the ionosphere, Rev. Geophys. Space Phys., 15, 113-127, 1977.

Pines, D. and Schrieffer, J. R.: Collective Behavior in Solid State Plasmas, Phys. Rev., 124, 1387-1400, 1961.

Robinson, R. M., Winningham, J. D., Sharber, J. R., Barch, J. L., and Heelis, R.: Plasma and field properties of suprathermal electron bursts, J. Geophys. Res., 94, 12 031-12 036, 1989.

Ronnmark, K.: Electron acceleration in the auroral current circuit, Geophys. Res. Lett., 26, 983-986, 1999.

Ronnmark, K. and Hamrin, M.: Auroral electron acceleration by
Alfven waves and electrostatic field, J. Geophys. Res., 105, 25 333-25 344, 2000

Roth, I. and Temerin, M.: Enrichment of ${ }^{3} \mathrm{H}_{e}$ and heavy ions in impulsive solar flares, Astro. J., 477, 940-957, 1997.

Saito, H., Yoshino, T., and Sato, N.: Narrow-banded ELF emissions over the southern Polar Region, Planet. Space Sci., 35, 745-752, 1987.

Scudder, J., Hunscker, F., Miller, G., Lobell, J., et al.: Hydra-A three-dimensional electron and ion hot plasma instrument for the polar Spacecraft of the GGS mission, Space Sci. Rev., 71, 459495, 1995.

Sharp, R. D., Johnson, R. G., and Shelley, E. G.: Observation of an ionospheric acceleration mechanism producing energetic $(\mathrm{keV})$ ions primarily normal to the geomagnetic field direction., J. Geophys. Res., 82, 3324-3328, 1977.

Smith-Mott H. M. and Langmuir, I.: The theory of collectors in gaseous discharges, Phys. Rev. , 28, 727-763, 1926.

Strangeway, R. J., Kepko, L., Elphic, R. C., Carlson, C. W., Ergun, R. E., McFadden, J. P., Peria, W. J., Delory, G. T., Chaston, C. C., Temerin, M., Cattell, C. A., Mobius, E., Kistler, L. M., Klumpar, D. M., Peterson, W. K., Shelley, E. G., and Pfaff, R. E.: FAST observations of VLF waves in the auroral zone: evidence of very low plasma densities, Geophys. Res. Lett., 25, 2065-2068, 1998.

Summers, D. and Thorne, R. M.: Plasma microinstabilities driven by loss-cone distributions, J. Plasma Phys., 53, 293-315, 1995.

Temerin, M. and Lysak, R. L.: Electromagnetic ion cyclotron (ELF) waves generated by auroral electron precipitation, J. Geophys. Res., 89, 2849-2859, 1984.

Temerin, M. and Roth, I.: The production of $3 \mathrm{He}$ and heavy ion enrichment in 3He-rich flares by electromagnetic hydrogen cyclotron waves, Astrophys. J., 391, 105-108, 1992.

Terashima, Y.: Particle Aspects Analysis of Drift Instability, Progr. Theore. Phys., 37, 775-785, 1967.

Thompson, B. J. and Lysak, R. L.: Electron acceleration by inertial Alfven waves, J. Geophys. Res., 101, 5359-5370, 1996.

Thorne, R. M. and Horne, R. B.: Energy transfer between energetic ring current $\mathrm{H}^{+}$and $\mathrm{O}^{+}$by electromagnetic ion cyclotron waves, J. Geophys. Res., 99, 17 275-17 282, 1994.

Thorne, R. M. and Horne, R. B.: Modulation of electromagnetic ion cyclotron instability due to interaction with ring current o' during magnetic storms, J. Geophys. Res., 102, 14 155-14 163, 1997.

Tiwari, M. S. and Rostoker, G.: Field aligned current and aurroral acceleration by non-linear MHD waves, Planet. Space Sci., 32, 1497-1503, 1984.

Tiwari, M. S. and Varma, P.: Drift instability in the presence of a parallel electric field and an inhomogeneous magnetic field: Particle aspect analysis, J. Plasma Phys., 46, 49-62, 1991.

Tiwari, M. S. and Varma, P.: Drift wave instability with loss-cone distribution function - particle aspect analysis, Planet. Space Sci., 41, 199-207, 1993.

Varma, P. and Tiwari, M. S.: Drift wave in the presence of inhomogeneous electric field with different distribution functionsparticle aspect analysis, Physica Scr., 44, 296-303, 1991.

Varma, P. and Tiwari, M. S.: Ion and electron beam effects on drift wave instability with different distribution functions-particle aspect analysis, Physica Scr., 45, 275-279, 1992.

Varma, P. and Tiwari, M. S.: Drift wave in the presence of an ac electric field with different distribution functions: Particle aspect analysis, Ind. J. Pure Appl. Phys., 31, 616-623, 1993. 
Wentworth, R. C., MacDonald, W. M., and Singer, S. F: Lifetimes of trapped radiation belt particles determind by coulomb scattering, Phys. Fluids., 2, 499-509, 1959.

Whalen, B. A., Bernstein, W., and Daly, P. W.: Low altitude acceleration of ionospheric Ions, Geophys. Res. Lett., 55-58, 1978.
Whipple Jr., E. C.: The signature of parallel electric fields in a collisionless plasma, J. Geophys. Res., 82, 1525-1531, 1977.

Yau, A. W., Whalen, B. A., McNamara, A. G., Kellogg, P. J., and Bernstein, W.: Particle and wave observations of low altitude ionospheric ion acceleration events, J. Geophys. Res., 88, 341$355,1983$. 